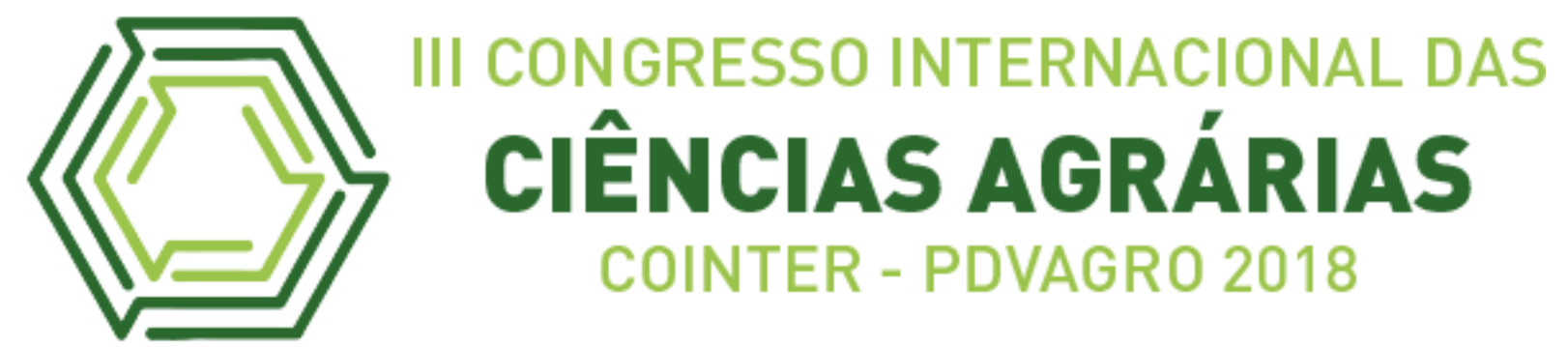

\title{
CARACTERIZAÇÃO FISICO QUÍMICA DE NEOSSOLOS EM AGROECOSSISTEMAS, MARTINS/RN
}

\section{CHEMICAL PHYSICAL CHARACTERIZATION OF NEOSOLS IN AGROECOSSISTEMAS, MARTINS / RN \\ Apresentação: Pôster}

\begin{abstract}
Phâmella Kalliny Pereira Farias ${ }^{1}$; Jeane Cruz Portela ${ }^{2}$; Thamirys Duarte Ávila ${ }^{3}$; Joseane Dunga da Costa ${ }^{4}$; Thais Cristina de Souza Lopes ${ }^{5}$
\end{abstract}

\section{DOI: https://doi.org/10.31692/2526-7701.IIICOINTERPDVAGRO.2018.00368}

\section{Introdução}

O manejo inadequado do solo e dos cultivos agrícolas, pode torná-lo esgotável, para que isso não ocorra é necessário determinar o uso mais racional da terra através do estudo dos diversos atributos envolvidos, realizado mediante um levantamento físico e químico. As diversas interrelações existentes entre os atributos do solo controlam os processos de sua variação ao longo do tempo e do espeço, sendo assim, qualquer alteração nessas relações pode interferir diretamente na sua estrutura (Cunha et al., 2012).

A fragilidade das áreas com Neossolos associada à falta de informações detalhadas sobre as características responsáveis pelo comportamento dos solos, justificam esforços no sentido de se entender melhor essa classe de solos. Nesse contexto, o objetivo do trabalho foi realizar uma caracterização físico química de Neossolos em agroecossistemas do Sítio Poção localizado no município de Martins/RN, utilizando como ferramenta para interpretação dos resultados a análise multivariada.

\section{Fundamentação Teórica}

No estado natural, o solo apresenta variabilidade nos seus atributos físicos e químicos

\footnotetext{
1 Programa de Pós-Graduação em Manejo de Solo e Água, Universidade Federal Rural do Semi-Árido, phamellakalliny@hotmail.com

${ }^{2}$ Doutora, Universidade Federal Rural do Semi-Árido, jeaneportela@ufersa.edu.br

${ }^{3}$ Engenharia Florestal, Universidade Federal Rural do Semi-Árido, thamysh_past@hotmail.com

${ }^{4}$ Programa de Pós-Graduação em Manejo de Solo e Água, Universidade Federal Rural do Semi-Árido, joseany_costa@hotmail.com

${ }^{5}$ Programa de Pós-Graduação em Manejo de Solo e Água, Universidade Federal Rural do Semi-Árido, thaiscristina13@hotmail.com
} 
em decorrência dos processos de formação ocorridos. Essa variabilidade pode ser influenciada pelos fatores de formação (material de origem, relevo, clima, organismos e tempo) e também pela ação antrópica, através das práticas de manejo (Carvalho et al., 2003)

Geralmente é dada uma maior importância apenas a camada superficial ou arável, desconhecendo o que está abaixo dos primeiros centímetros da superfície, o que contribui para uma forma de manejo inadequada (Guerra e Cunha, 2004). Entender o processo de formação do solo é essencial para sua melhor utilização, evitando o esgotamento.

\section{Metodologia}

A pesquisa e coleta de solos foram realizadas na área do perímetro do Sítio Poção, no município de Martins, no estado do Rio Grande do Norte, com coordenadas geográficas: $6^{\circ} 05^{\prime}$ $16^{\prime \prime}$ de latitude Sul e $37^{\circ} 54^{\prime} 40^{\prime \prime}$ de longitude Oeste. Foram selecionados três perfis com coletas realizadas segundo o Sistema Brasileiro de Classificação de solos (Santos et al., 2013). As análises físicas foram realizadas (três repetições da mesma amostra) e constaram de: granulometria, densidade do solo (na terra fina seca ao ar TFSA) e concentração de cascalho, todas conforme Teixeira et al. (2017). As análises químicas foram realizadas utilizando-se a amostra na forma de TFSA, também em três repetições, de acordo com os métodos descritos em Teixeira, et al. (2017) e constaram de: $\mathrm{pH}$ em água; $\mathrm{P}$ disponível, $\mathrm{Na}^{+}$e $\mathrm{K}^{+} ; \mathrm{Ca}^{2+}, \mathrm{Mg}^{2+} \mathrm{e}$ $\mathrm{Al}^{3+} ;(\mathrm{H}+\mathrm{Al})$. O carbono orgânico total (COT) foi determinado por oxidação via úmida utilizando-se dicromato de potássio 0,167 mol L-1 (Yeomans \& Bremner, 1988). A partir das análises realizadas foram obtidos os índices: soma de bases (SB); capacidade de troca catiônica efetiva (t); capacidade de troca catiônica a pH 7,0 (CTC); saturação por bases (V \%); saturação por alumínio trocável (m \%) e a percentagem de sódio trocável (PST). Sendo determinados segundo o manual de métodos de análises de solo da Embrapa (Teixeira et al., 2017). Para a distinção dos perfis de solo, foram empregadas técnicas de análise multivariada, em especial pela correlação de Pearson, Análise Fatorial e Análise de Componentes Principais - ACP, utilizando o software Statistica 7.0 (StatSoft, 2004), tendo como variáveis os atributos físicos e químicos do solo. Os resultados das análises químicas foram interpretados conforme Manual de Recomendações para uso de corretivos e fertilizantes em Minas Gerais (Ribeiro, Guimarães, Venegas,1999).

\section{Resultados e Discussões}


Na composição granulométrica dos perfis estudados, observa-se que os maiores teores de argila encontrados foram no perfil 1, oscilando entre 170 e $260 \mathrm{~g} / \mathrm{kg}$, isso ocorreu porque a área apresenta uma deposição de sedimento (Tabela 1).

Tabela 1. Atributos físicos dos perfis de solo selecionados no Sítio Poção, Martins/RN.

\begin{tabular}{|c|c|c|c|c|c|c|}
\hline \multirow{2}{*}{$\begin{array}{c}\text { Hor./Prof. } \\
\text { cm }\end{array}$} & \multicolumn{3}{|c|}{ Granulometria (g/kg) } & \multirow{2}{*}{$\begin{array}{c}\text { Classe textural } \\
\text { SiBCS } \\
\end{array}$} & \multirow{2}{*}{$\begin{array}{c}\text { Ds } \\
\left(\mathrm{g} / \mathrm{cm}^{-3}\right)\end{array}$} & \multirow{2}{*}{$\begin{array}{c}\text { Cascalho } \\
\%\end{array}$} \\
\hline & Areia Total & Silte & Argila & & & \\
\hline \multicolumn{7}{|c|}{ Perfil 1- Associação NEOSSOLO FLÚVICO/LUVISSOLO } \\
\hline $\mathrm{A}(0-15)$ & 400 & 340 & 260 & Franco & 1,20 & 1,2 \\
\hline $1 \mathrm{C}(15-34)$ & 600 & 210 & 190 & Franco Arenosa & 1,41 & 4,3 \\
\hline $2 \mathrm{C}(34-55)$ & 620 & 210 & 170 & Franco Arenosa & 1,60 & 1,3 \\
\hline $\mathrm{AL}(55-65)$ & 620 & 180 & 200 & Franco Arenosa & 1,82 & 2,4 \\
\hline $\mathrm{B}(65-85)$ & 620 & 150 & 230 & Franco Arenosa & 1,73 & 3,0 \\
\hline \multicolumn{7}{|c|}{ Perfil 2-Associação NEOSSOLO LITÓLICO/NEOSSOLO FLÚVICO } \\
\hline $\mathrm{A}_{\mathrm{FLU}}(0-10)$ & 380 & 390 & 230 & Franco & 1,18 & 5,5 \\
\hline $\mathrm{C}_{\mathrm{FLU}}(10-20)$ & 630 & 210 & 160 & Franco Arenosa & 1,61 & 8,5 \\
\hline $\mathrm{A}_{\mathrm{NEO}}(20-50)$ & 630 & 180 & 190 & Franco Arenosa & 1,27 & 13,2 \\
\hline \multicolumn{7}{|c|}{ Perfil 3- NEOSSOLO LITÓLICO } \\
\hline $\mathrm{A}(0-28)$ & 630 & 240 & 130 & Franco Arenosa & 1,22 & 10,4 \\
\hline $\mathrm{A}_{\mathrm{COL}}(28-44)$ & 640 & 210 & 150 & Franco Arenosa & 1,18 & 10,8 \\
\hline$C_{R}(44-90)$ & 700 & 150 & 150 & Franco Arenosa & 1,24 & 14,0 \\
\hline
\end{tabular}

A fração silte apresentou um decréscimo em profundidade, variando de 150 a 390 g/kg, esse fato pode ser explicado pela ação dos agentes ativos durante o processo de intemperismo. Os valores de densidade dos solos variaram de 1,18 a $1,82 \mathrm{~g} / \mathrm{cm}^{-3}$, expressando uma restrição para o desenvolvimento de atividades agrícolas. Os altos valores de Ds estão relacionados a predominância da fração areia, que juntamente com a fração silte, indicam reservas nutricionais.

Os solos em estudo apresentam reação ácida com predominância de cargas positivas e apresentando limitações químicas (Tabela 2).

Tabela 2. Atributos químicos dos perfis de solo selecionados no Sítio Poção, Martins/RN

\begin{tabular}{|c|c|c|c|c|c|c|c|c|c|c|c|c|c|c|c|c|c|}
\hline Hor. & COT & pH & $\mathbf{C E}$ & $\mathbf{P}$ & $\mathbf{K}^{+}$ & $\mathbf{N a}^{+}$ & $\mathbf{C a}^{2+}$ & $\mathbf{M g}^{2+}$ & $\mathbf{A l}^{3+}$ & $(\mathbf{H}+\mathbf{A l})$ & SB & $\mathbf{t}$ & CTC & \multirow{2}{*}{$\begin{array}{c}\text { ATA } \\
\text { cmolc/kg } \\
\text { de argila }\end{array}$} & $\mathbf{V}$ & $\mathbf{m}$ & PST \\
\hline & $\mathrm{g} / \mathrm{kg}$ & & $\mathrm{dS} / \mathrm{m}$ & $\mathrm{mg} / \mathrm{kg}$ & \multicolumn{9}{|c|}{$\mathrm{cmolc} / \mathrm{kg}$} & & & $\%$ & \\
\hline \multicolumn{18}{|c|}{ Perfil 1- Associação NEOSSOLO FLÚVICO/LUVISSOLO } \\
\hline A & 34,63 & 4,32 & 1,37 & 30,68 & 1,09 & 3,44 & 7,77 & 3,72 & 0,29 & 5,84 & 13,53 & 13,82 & 19,36 & 72,44 & 69,86 & 2,10 & 0,08 \\
\hline $1 \mathrm{C}$ & 17,91 & 3,98 & 0,23 & 55,34 & 0,21 & 3,37 & 4,15 & 1,25 & 0,71 & 4,88 & 7,40 & 8,11 & 12,28 & 63,22 & 60,24 & 8,76 & 0,12 \\
\hline $2 \mathrm{C}$ & 16,72 & 4,30 & 0,20 & 83,80 & 0,13 & 4,03 & 5,02 & 2,04 & 0,49 & 2,63 & 9,44 & 9,93 & 12,07 & 70,46 & 78,18 & 4,93 & 0,15 \\
\hline$A_{L}$ & 10,77 & 4,74 & 0,20 & 77,30 & 0,30 & 2,78 & 5,91 & 1,84 & 0,29 & 1,91 & 9,40 & 9,69 & 11,32 & 56,08 & 83,07 & 2,96 & 0,11 \\
\hline
\end{tabular}




\begin{tabular}{|c|c|c|c|c|c|c|c|c|c|c|c|c|c|c|c|c|c|}
\hline B & 7,95 & 5,03 & 0,23 & 62,06 & 0,25 & 2,84 & 6,18 & 1,98 & 0,21 & 1,31 & 9,85 & 10,05 & 11,16 & 48,91 & 88,26 & 2,06 & 0,11 \\
\hline \multicolumn{18}{|c|}{ Perfil 2-Associação NEOSSOLO LITÓLICO/NEOSSOLO FLÚVICO } \\
\hline $\mathrm{A}_{\mathrm{FLU}}$ & 45,73 & 6,40 & 1,42 & 186,24 & 3,00 & 5,82 & 11,07 & 5,08 & 0,00 & 1,19 & 19,60 & 19,60 & 20,79 & 89,26 & 94,28 & 0,00 & 0,12 \\
\hline $\mathrm{C}_{\mathrm{FLU}}$ & 21,53 & 4,87 & 0,42 & 149,03 & 0,65 & 2,91 & 6,56 & 2,14 & 0,18 & 2,04 & 10,42 & 10,61 & 12,46 & 80,76 & 83,67 & 1,73 & 0,10 \\
\hline $\mathrm{A}_{\mathrm{NEO}}$ & 5,83 & 5,22 & 0,74 & 100,84 & 0,41 & 3,62 & 8,15 & 2,63 & 0,14 & 1,47 & 12,93 & 13,07 & 14,39 & 75,10 & 89,78 & 1,08 & 0,11 \\
\hline \multicolumn{18}{|c|}{ Perfil 3- NEOSSOLO LITÓLICO } \\
\hline A & 43,74 & 6,35 & 0,32 & 233,19 & 0,72 & 0,41 & 9,81 & 0,83 & 0,10 & 1,43 & 10,88 & 10,99 & 12,31 & 95,64 & 88,38 & 0,94 & 0,01 \\
\hline $\mathrm{A}_{\mathrm{COL}}$ & 22,68 & 6,17 & 0,31 & 170,93 & 0,42 & 0,74 & 6,68 & 3,43 & 0,00 & 1,40 & 10,54 & 10,55 & 11,95 & 81,73 & 88,25 & 0,03 & 0,03 \\
\hline $\mathrm{C}_{\mathrm{R}}$ & 9,54 & 5,80 & 0,28 & 83,02 & 0,20 & 0,27 & 5,08 & 2,28 & 0,00 & 1,55 & 7,52 & 7,53 & 9,08 & 59,19 & 82,76 & 0,05 & 0,01 \\
\hline
\end{tabular}

Hor. - horizonte; $\mathrm{pH}$ - potencial hidrogeniônico; $\mathrm{CE}$ - condutividade elétrica; $\mathrm{P}$ - fósforo; COT - carbono orgânico total; $\mathrm{Ca}^{2+}$ cálcio; $\mathrm{Mg}^{2+}$ - magnésio; $\mathrm{K}^{+}$- potássio; $\mathrm{Na}^{+}$- sódio; $\mathrm{Al}^{3+}$ - alumínio; $(\mathrm{H}+\mathrm{Al})$ - acidez potencial; $\mathrm{SB}$ - soma de bases; $\mathrm{t}$ capacidade troca catiônica efetiva; CTC - capacidade de troca catiônica potencial; $\mathrm{V}$ - saturação por bases; $\mathrm{m}$ - saturação por alumínio; PST - porcentagem de sódio trocável

O maior teor de condutividade elétrica $(1,42 \mathrm{dS} / \mathrm{m})$ foi encontrado no horizonte superficial do Perfil 2, este maior valor deve-se ao aumento da concentração de sódio trocável no solo $(5,82 \mathrm{cmolc} / \mathrm{kg})$. Todos os perfis apresentaram caráter eutrófico $(\mathrm{V}>50 \%)$, tal fato está relacionado aos teores de $\mathrm{Na}+$, que superestimam a fertilidade, sendo fator limitante ao crescimento das plantas.

Para o carbono orgânico total (COT), os maiores teores foram encontrados nos horizontes superficiais, variando de $34,63 \mathrm{~g} / \mathrm{kg}$ a $45,73 \mathrm{~g} / \mathrm{kg}$, decrescendo com a profundidade. Os altos teores de fósforo encontrados podem estar relacionados a atividade antrópica existente, já que o manejo adotado nas áreas com preparo mínimo e preservação dos resíduos na superfície contribui para a manutenção da matéria orgânica que funciona como fonte de fósforo.

De acordo com as cargas fatoriais dos atributos foi obtido como principais resultados os fatores 1, 2, 3 e 4 os quais explicaram 96,06\% da variação dos dados (Tabela 3).

Tabela 3 - Cargas fatoriais dos atributos químicos dos solos analisados e seus respectivos Autovalores, variâncias totais observadas e acumuladas.

\begin{tabular}{|c|c|c|c|c|}
\hline \multirow[b]{3}{*}{ Variáveis } & \multicolumn{4}{|c|}{ Cargas fatoriais ${ }^{(1)}$} \\
\hline & Fator $1^{(2)}$ & Fator $2^{(2)}$ & Fator $3^{(2)}$ & Fator $4^{(2)}$ \\
\hline & $\begin{array}{c}\text { Disponibilidade } \\
\text { De nutriente }\end{array}$ & $\begin{array}{c}\text { Acidez do } \\
\text { solo } \\
\end{array}$ & $\begin{array}{c}\text { Disponibilidade } \\
\text { de fósforo }\end{array}$ & $\begin{array}{c}\text { Disponibilidade } \\
\text { de sódio }\end{array}$ \\
\hline COT & 0,59 & $-0,04$ & 0,72 & 0,21 \\
\hline $\mathrm{pH}$ & 0,09 & 0,77 & 0,43 & 0,35 \\
\hline $\mathrm{CE}$ & $\mathbf{0 , 9 7}$ & 0,09 & 0,03 & 0,11 \\
\hline $\mathrm{P}$ & $-0,02$ & 0,51 & $\mathbf{0 , 8 5}$ & 0,12 \\
\hline $\mathrm{K}^{+}$ & $\mathbf{0 , 8 4}$ & 0,26 & 0,36 & $-0,09$ \\
\hline $\mathrm{Na}^{+}$ & 0,66 & $-0,17$ & $-0,04$ & $-0,68$ \\
\hline $\mathrm{Ca}^{2+}$ & 0,62 & 0,49 & 0,52 & 0,05 \\
\hline $\mathrm{Mg}^{2+}$ & $\mathbf{0 , 8 4}$ & 0,34 & $-0,11$ & 0,06 \\
\hline $\mathrm{Al}^{3+}$ & $-0,16$ & $-0,91$ & $-0,08$ & $-0,35$ \\
\hline
\end{tabular}




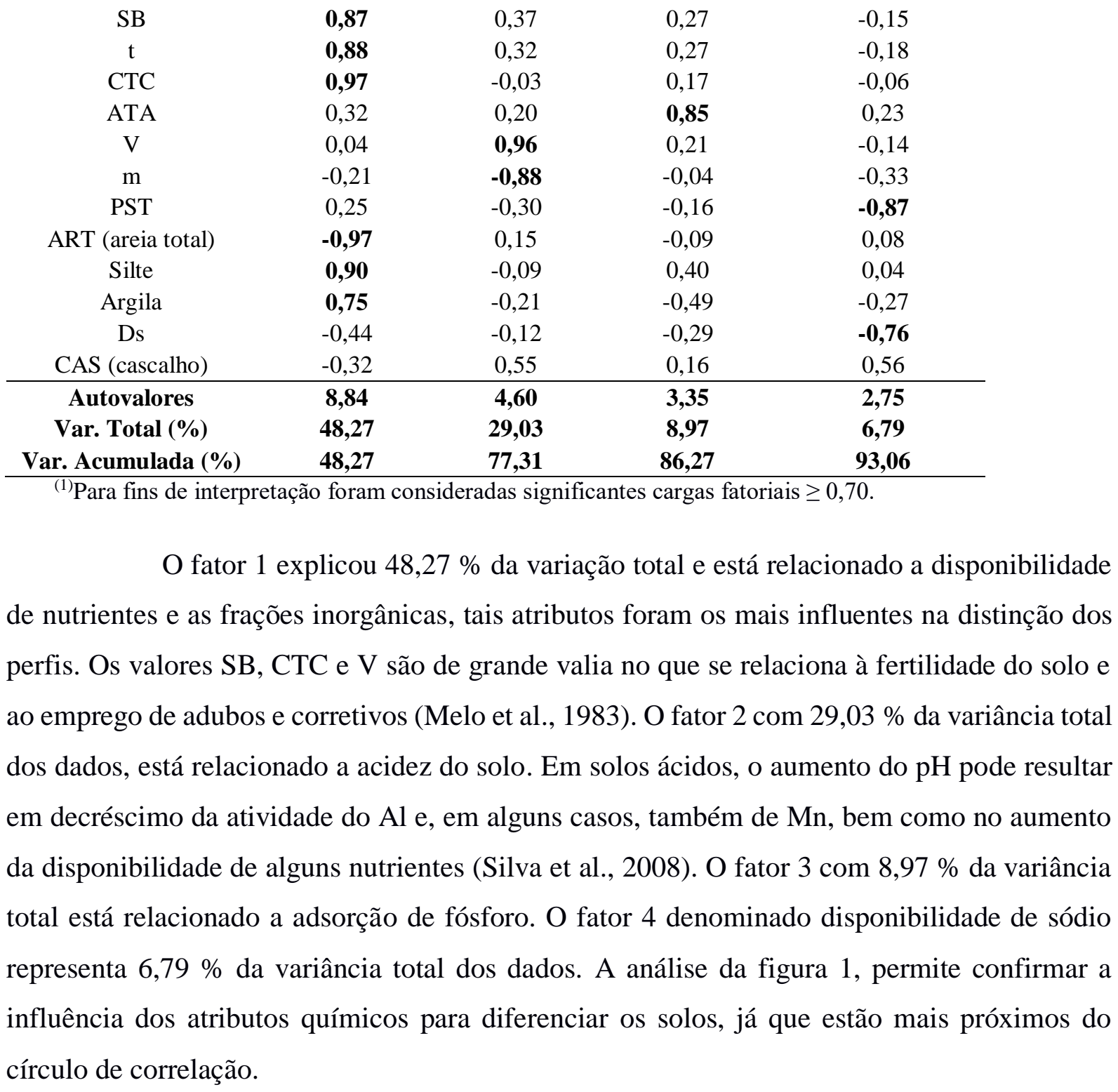

Figura 1. Projeção dos vetores dos atributos do solo dos perfis estudados. 

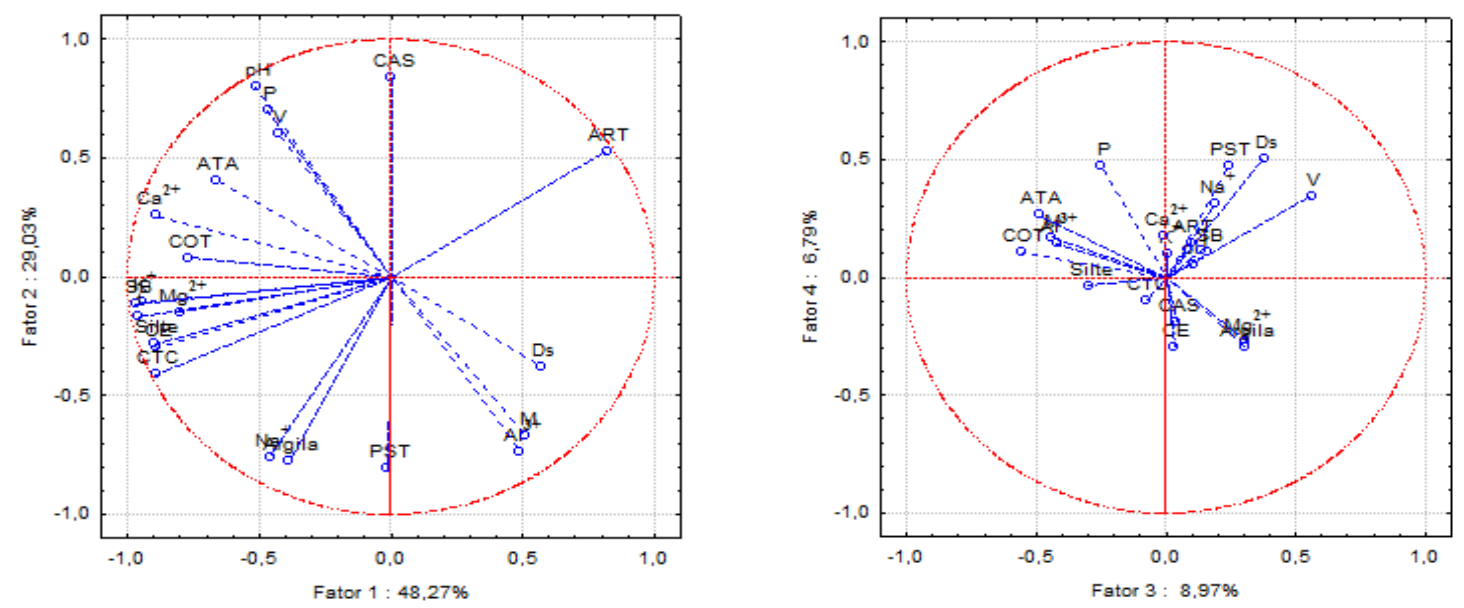

\section{Conclusões}

O manejo adotado nos solos das áreas de estudo favoreceu suas boas reservas de $\mathrm{P}$ através da manutenção dos resíduos na superfície, mas apresentam limitações químicas relacionadas a $\mathrm{CE}, \mathrm{pH}$ e $\mathrm{V}$ (superestimada pelos teores de $\mathrm{Na}^{+}$).

Os fatores químicos $\mathrm{CE}, \mathrm{K}^{+}, \mathrm{Mg}^{2+}, \mathrm{SB}, \mathrm{t}, \mathrm{CTC}$ e os fatores físicos ART, Silte e Argila foram os mais sensíveis na diferenciação dos solos, indicando que as reservas de nutrientes estão associadas também as frações inorgânicas.

\section{Referências}

Carvalho, M. P; Takeda, E. Y; Freddi, O. S. Variabilidade espacial de atributos de um solo sob videira em Vitória Brasil (SP). Revista Brasileira Ciência do Solo. Viçosa - MG. n.27. 2003.

Cunha, E.Q. et al. Atributos físicos, químicos e biológicos de solo sob produção orgânica impactados por sistemas de cultivo. Revista Brasileira de Engenharia Agrícola e Ambiental, Campina Grande, v. 16, n. 1, p. 56-63, 2012.

Ernani, P. R. Química do solo e disponibilidade de nutrientes. Lages: UDESC. 2008. 230p.

Guerra, A. J. T.; Cunha, S. B. Geomorfologia e Meio Ambiente. Rio de Janeiro: Bertrand Brasil, p.68- 70, 2004

Melo, F. de A.F. de et al. Fertilidade do solo. 1. ed. São Paulo: Nobel, 1983. 400 p.

Ribeiro, A.C.; Guimarães, P.T.G.; Alvarez Venegas, V.H. Recomendações para uso de corretivos e fertilizantes em Minas Gerais, 5a aproximação. Viçosa: SFSEMG, 1999. 359p.

Santos, R. D. et al. Manual de descrição e coleta de solo no campo. 6.ed. Viçosa: Sociedade Brasileira de Ciência do Solo, 2013. 100 p 
Silva, I.R. e Mendonça, E.S. Matéria Orgânica Do Solo. In:Novais, R.F.; Alvarez V., V.H.; Barros, N.F.;Fontes, R.L.F.; Cantarutti, R.B. \& Neves, J.C.L. Fertilidade Do Solo. 1. Ed. Viçosa: Sociedade Brasileira De Ciência Do Solo, 2008. P.275-374.

StatSoft, Inc. (2004). STATISTICA (data analysis software system), version 7. www.statsoft.com.

Texeira, P. C. et al. Manual de métodos de análise de solos. 3.ed. rev. e ampl. Brasília, DF: Embrapa Solos, 2017. 573 p.

Yeomans, J. C. \& Bremner, J. M. A rapid and precise method for routine determination of carbon in soil. Communications in Soil Science Plant Analysis, 19: 1467-1476, 1988. 